\title{
Clinical application for DSA combined with a double-chamber Fogarty catheter in the treatment of venous crisis
}

\author{
Xiaodong Li, Xiaowei Yan, Haijun Jiang, Rui Gu, Qiang Xie, Changyu Yu, Pei Wang \\ Department of Hand and Foot Surgery, Affiliated Hospital of Chengde Medical College, Chengde City, China
}

Videosurgery Miniinv 2021; 16 (2): 347-354

DOI: https://doi.org/10.5114/wiitm.2020.101231

\begin{abstract}
Introduction: Venous crisis, as a common vascular crisis post limb replantation, is usually treated with surgical exploration.

Aim: To investigate effects of digital subtraction angiography (DSA) combined with double-chamber Fogarty balloon catheter on venous crisis post replantation of limbs.

Material and methods: Twelve patients suffering from severed limbs were involved in this study. Patients underwent DSA combining double-chamber Fogarty balloon catheter operation. Colour Doppler ultrasound was used to diagnose patients with venous crisis. Patients were treated with rehydration, anti-infection, anticoagulation, and vasodilation. Indexes, including total joint active activity, working condition, remaining symptoms, appearance, feeling, and muscle strength, were evaluated.

Results: During operation, the limb was shortened to $0-1 \mathrm{~cm}$ in 8 cases, to $1-2 \mathrm{~cm}$ in 2 cases, and to $2-2.5 \mathrm{~cm}$ in 2 cases. According to DSA findings, popliteal vein thrombosis was formed at $0.6-4.2 \mathrm{~cm}$ and was removed from the popliteal vein. After removal of the thrombosis, DSA images showed re-canalization of the popliteal vein. A typical case of a 16-year-old patient underwent limb replantation; however, venous crisis was formed post operation. Postoperative colour Doppler ultrasound findings indicated re-canalization of the popliteal vein. Tibia and fibula were reduced and internally fixed, while the limb was survived post-operation. The degree of swelling of limbs was improved, and skin temperature was normal or $0.6-1.5^{\circ} \mathrm{C}$ lower than affected limbs. Skin colour was normal and activity was improved. Patients demonstrated sensory recovery grade of $\mathrm{S}^{3+}$ and two-point discrimination of $4.5 \mathrm{~mm}$. Conclusions: DSA combining double-chamber Fogarty balloon catheter, as a minimally invasive and fast approach, could accurately locate thrombi and improve pertinence of vein branches.
\end{abstract}

Key words: digital subtraction angiography, double-chamber Fogarty balloon catheter, limb replantation, severed limbs, venous crisis.

\section{Introduction}

With the rapid development of high-speed transportation and heavy industry, the incidence rate of severed limbs is increasing year by year [1]. Limb replantation has been proven to be an effective strategy for treating severed limbs [2]. Because of the special physiological structure of veins, such as thin wall, low pressure, poor elasticity, and slow flow rate, venous crisis is the common vascular crisis after limb replantation, which often leads to limb necrosis, amputation, and even death [3].

Nowadays, treatment of venous crisis is mainly through surgical exploration [4]. The traditional open-exploration operation can only improve the anastomotic condition of blood vessels; however, it

\section{Address for correspondence}

Prof. Pei Wang, Department of Hand and Foot Surgery, Affiliated Hospital of Chengde Medical College, Chengde City, China,

e-mail: ckgkwp@163.com 
cannot deal with the distal lesions of blood vessels [5]. The traditional open-exploration operation may also cause secondary trauma to the repair area of the broken end, the effect of which on the combined thrombus and non-anastomotic thrombus is poor $[6,7]$. The double-chamber Fogarty balloon catheter, as a device for thrombectomy and thrombolysis, is widely used in the diagnosis and treatment of venous crisis [8]. Digital subtraction angiography (DSA) is often used in the diagnosis and treatment of venous crisis [9].

In recent years, DSA combined with a double-chamber Fogarty balloon catheter for treating thrombectomy is mainly used in patients with acute and chronic thrombus caused by basic cardiovascular disease [10, 11]. However, DSA combined with a double-chamber Fogarty balloon catheter is rarely reported for the treatment of venous crisis after

Table I. General information of patients

\begin{tabular}{|c|c|}
\hline Characteristics & Values \\
\hline \multicolumn{2}{|l|}{ Gender, $n$ : } \\
\hline Male & 7 \\
\hline Female & 5 \\
\hline Age, mean (range) [years] & $35(20-55)$ \\
\hline \multicolumn{2}{|l|}{ Cause of disease: } \\
\hline Tamp injury & 5 \\
\hline Incised wound & 2 \\
\hline Cut wound & 2 \\
\hline Explosive injury & 1 \\
\hline Crush injury & 2 \\
\hline \multicolumn{2}{|l|}{ Disarticulation region, $n$ : } \\
\hline Knee disarticulation & 2 \\
\hline Shin disarticulation & 6 \\
\hline Ankle joint disarticulation & 4 \\
\hline \multicolumn{2}{|l|}{ Disarticulation degree, $n$ : } \\
\hline Complete disarticulation & 7 \\
\hline Incomplete disarticulation & 5 \\
\hline Onset to hospital [h] & $6.5(1.5-14)$ \\
\hline Severed limbs to replantation [h] & $13.5(6.5-20.5)$ \\
\hline Occurrence of venous crisis [h] & $11.2(3-18)$ \\
\hline
\end{tabular}

the replantation of limbs. From June 2011 to January 2016, the DSA combined with double-chamber Fogarty balloon catheter was applied to treat 12 patients with venous crisis after replantation of limbs, with satisfactory effect, in this study.

This study aimed to investigate the effects of DSA combined with double-chamber Fogarty balloon catheter on venous crisis after replantation of limbs.

\section{Material and methods}

\section{Patients}

This study involved a total of 12 patients suffering from severed limbs, from June 2011 to January 2016. All of the patients underwent DSA combined with double-chamber Fogarty balloon catheter operation. The general information is shown in Table I. All cases were replanted by doctors meeting the microsurgery access standard and were operated in an emergency setting.

\section{Ethical approval and consent to participate}

This study was approved by the Ethical Committee of the Affiliated Hospital of Chengde Medical College, Chengde, China. All patients provided written informed consent and approved this study.

\section{Preoperative preparation}

Colour Doppler ultrasound was used to diagnose patients with venous crisis. For the symptoms, including pain, cold, emotional changes, and body position changes, low molecular weight heparin sodium injection, urokinase injection, and other drugs were applied for conservative treatment. We also improved the examination of colour Doppler ultrasound, coagulation, D-dimer and electrolyte, and actively managed the peri-operative period for administration of the DSA combined with double-chamber Fogarty balloon catheter together with thrombolytic/anticoagulant drugs.

\section{Operation procedures}

After satisfactory anaesthesia, a longitudinal " $\mathrm{S}$ " incision, about $8 \mathrm{~cm}$ long, was made in the middle and lower part of the affected thigh. Paying attention to the protection of the superficial vein, the distal end of the femoral vein was revealed, which is about 4-5 cm long. The proximal end of the femoral vein was blocked by noninvasive forceps, and a lon- 
gitudinal incision was made on the anterior wall of the femoral vein, about 3-4 cm in length. Continuous infusion of heparin saline was performed, with the assistance of a cardiologist, carefully measured in vitro. An appropriate catheter and guide wire were placed, and continuous angiography was carried out to determine the site and quantity of thrombus. The catheter was removed, keeping the guide wire, according to the information provided by colour Doppler ultrasound from before the operation, carefully comparing the different length of catheter, and selecting a Fogarty balloon catheter with appropriate diameter. The balloon was carefully inserted into the lumen when it withered. The resistance of the catheter changed from large to small, indicating that the catheter passed through the thrombus. After injecting heparin saltwater slowly, the balloon expanded, and the water volume in the balloon was adjusted carefully, so that the water capsule and the vein lumen could be evenly attached. The thrombus was then completely removed by the pulling force of the water sac

The Fogarty balloon catheter was used as the "channel" to inject the saline containing urokinase and heparin into the vein and squeeze the leg from the bottom to the top. Attention should be paid to protecting the broken end of the affected limb to avoid side injury. It can be seen that the thrombus in the distal vein branch has been flushed out. When there is a large amount of blood outflow in the distal vein of the limb, it indicates that the thrombus in the distal vein is basically removed. The remaining thrombus was examined, and the effect of operation was evaluated. The above operations were carried out 2-3 times consecutively to avoid aggravating vascular intimal injury. Before closing the incision, the Fogarty balloon catheter was kept in for 15-30 min. Urokinase and heparin-containing saline were continuously injected into the catheter to dissolve the remaining thrombus and prevent the formation of thrombus again. Then the Fogarty balloon catheter was removed, the femoral vein was repaired, bandaged with sterile dressing and fixed with plaster. A graph showing DSA combined with a double-chamber Fogarty catheter operation is shown in Photo 1.

\section{Postoperative management}

The patients were treated with rehydration, antiinfection, anticoagulation, and vasodilation. Uroki- nase 100,000-200,000 U was intravenously dropped once a day for 8-10 days. Papaverine injection $30 \mathrm{mg}$ was intramuscularly injected once every $6 \mathrm{~h}$, $30 \mathrm{mg}$ per time. Low-molecular-weight dextran injection $(500 \mathrm{ml})$ was intravenously dropped once a day for 7 days. Low-molecular-weight heparin (5000 IU) was injected subcutaneously once or twice a day for 5 days. The limb was locally baked with the lamp irradiation $\left(25^{\circ} \mathrm{C}, 40 \mathrm{~cm}\right.$ away from the affected limb). The attentions must be paid to monitor the coagulation mechanism, biochemical items, vital signs and limb blood circulation.

\section{Observation index}

The therapeutic effect was evaluated according to the "Functional evaluation of replantation of severed limbs by the hand surgery branch of the Chinese Medical Association" [12]. In this study, we evaluated the following indexes: 1) Total joint active activity (30 points). 2) Working condition (10 points). 3) Remaining symptoms (10 points). 4) Appearance (10 points). 5) Feeling (20 points). 6) Muscle strength (20 points). The therapeutic effect was represented as the following grading standard: Poor (below 39 points), fair (40-59 points), good (60-79 points), and excellent (80-100) points.

\section{Results}

\section{Preoperative condition of patients}

Among the patients, there were 7 cases with complete disarticulation and 5 cases with incomplete disarticulation (Table I). Among the causes of severed limbs, there were 5 cases of tamp injury, 2 cases of incised wound, 2 cases of cut wound,

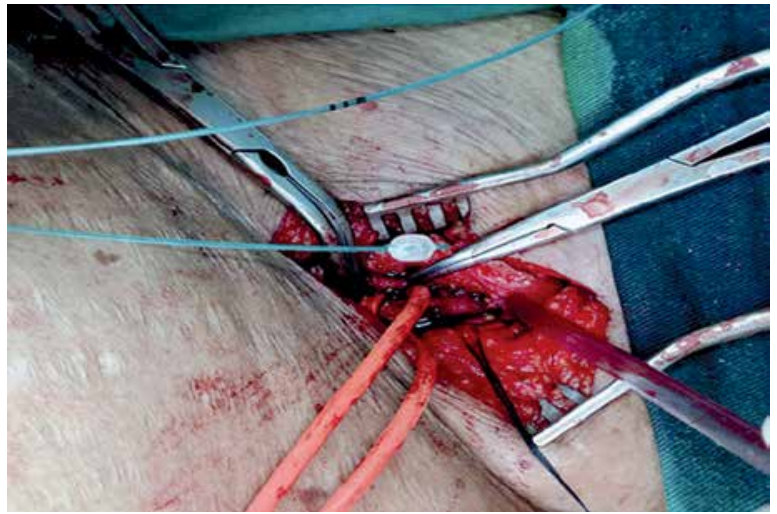

Photo 1. Graph for the DSA combining doublechamber Fogarty catheter operation 

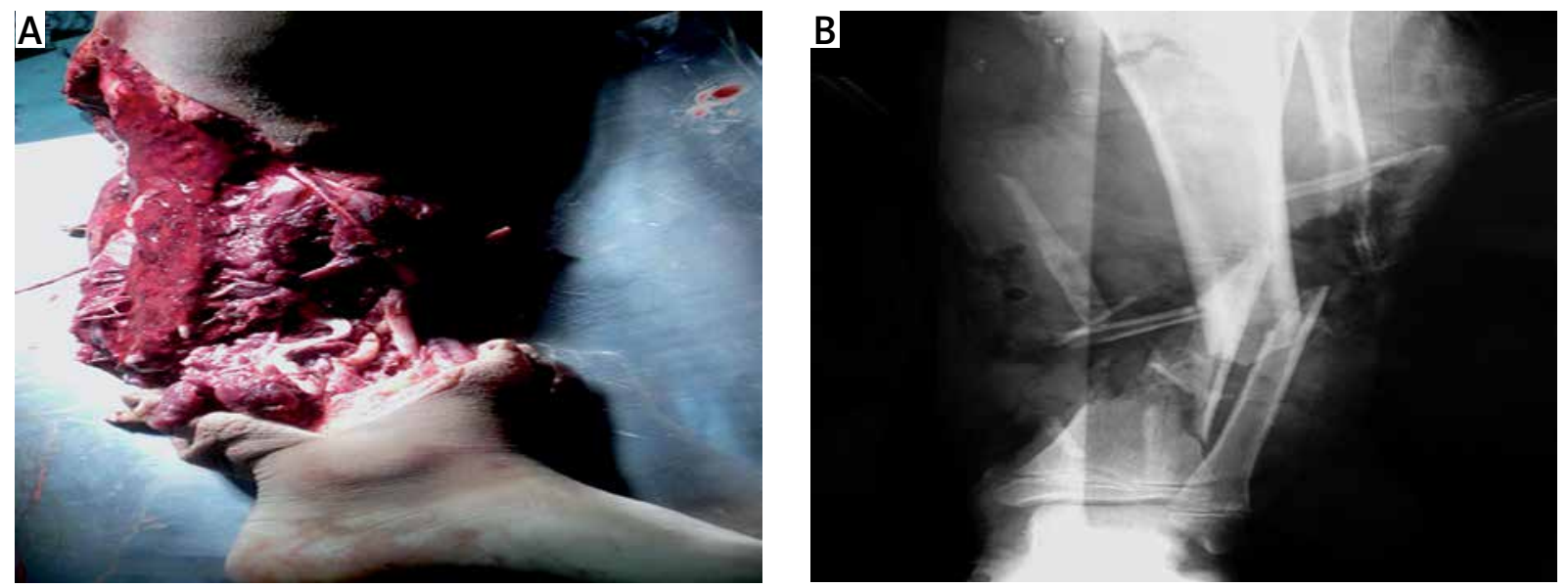

Photo 2. A typical case of a 16-year-old patient suffering from tamp injury. A - Image for tamp injury. B - X-ray examination demonstrating tibial and fibular fractures

1 case of explosive injury, and 2 cases of crush injury (Table I). All patients underwent limb replantation (DSA combined with double-chamber Fogarty balloon catheter method). A typical case of a 16-yearold patient suffering from tamp injury is illustrated in Photo $2 \mathrm{~A}$. The X-ray examination demonstrated tibial and fibular fractures (Photo $2 \mathrm{~B}$ ).

\section{Intra-operative condition of patients}

During the operation, the limb was shortened by $0-1 \mathrm{~cm}$ in 8 cases, to $1-2 \mathrm{~cm}$ in 2 cases, and to 2$2.5 \mathrm{~cm}$ in 2 cases. According to the DSA findings (Photo $3 \mathrm{~A}$ ), the popliteal vein thrombosis was formed (with length $0.6-4.2 \mathrm{~cm}$ ), and was successfully removed from the popliteal vein (Photo $3 \mathrm{~B}$ ). After the removal of thrombosis, the DSA image showed re-canalisation of the popliteal vein (Photo $3 \mathrm{C}$ ).

\section{Venous crisis manifestations}

The main clinical manifestations of postoperative venous crisis were demonstrated as follows: 1) The skin colour of the early affected limb was purple. 2) A tension blister formed. 3) The temperature of the limb decreased gradually. 4) The red/white reaction decreased. 5) The blood colour of the late acupuncture test was dark purple. 6) The red/white reaction was not obvious. 7) The skin tension increased. 8) The skin temperature was low. 9) There was sensory disturbance.

In a typical case of a 16-year-old patient who underwent limb replantation, however, the venous crisis was formed post-operation (Photo $4 \mathrm{~A}$ ), accord- ing to the colour Doppler ultrasound image. Then, the patients were administered with DSA combined with double-chamber Fogarty balloon catheter for carrying out the thrombolysis and anticoagulation. The postoperative colour Doppler ultrasound findings indicated re-canalisation of the popliteal vein (Photo 4 B).

\section{Postoperative condition of patients}

The postoperative $X$-ray findings of the above patient showed that the tibia and fibula were reduced and internally fixed (Photo $5 \mathrm{~A}$ ). Meanwhile, the limb was survived after the operation (Photo $5 \mathrm{~B}$ ).

There were 12 patients, all of whom were alive. The incision of 11 cases healed in stage I, among whom 1 case had osteofascial compartment syndrome, which recovered well. Among these 11 cases, 1 case of acute liver function damage was treated by internal medicine and was corrected. Moreover, among 12 cases, 1 case had vein crisis again after the operation, diagnosed by colour Doppler ultrasound. This vein crisis was anastomotic thrombus. Subsequently, incision and exploration were performed, and the vein thrombus appeared again, with necrotic limbs. Finally, amputation was performed and the incision healed in stage $I$.

\section{Follow-up}

Except for 1 case undergoing incision and exploration (finally illustrating venous crisis and conducted the amputation), a total of 11 patients were followed up in this study. The average follow-up period 

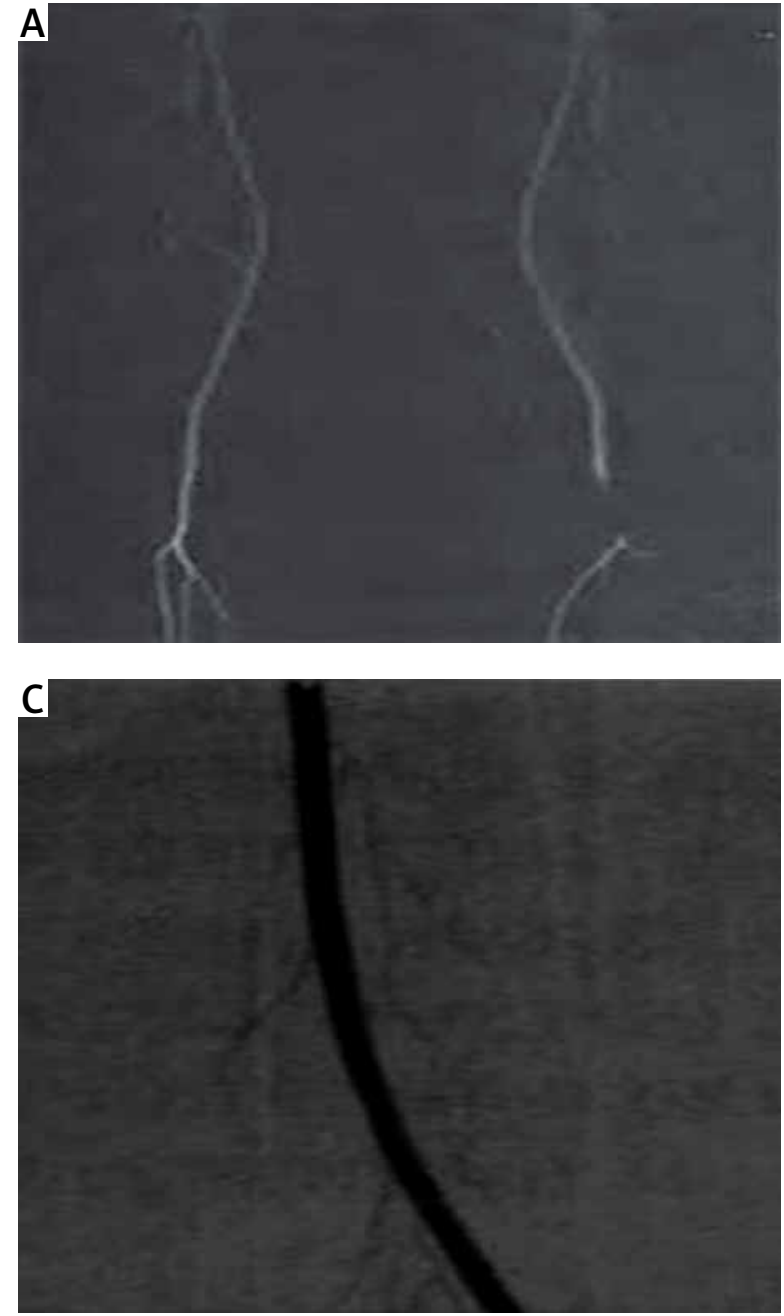

of all patients was 11 months (7-29 months). The shape of the affected limb was satisfactory, and the blood circulation of the limb was improved. Patients demonstrated capillary filling times of 1.5-3.3 s. The swelling degree of the limbs was improved, and the skin temperature was normal or $0.6-1.5^{\circ} \mathrm{C}$ lower than that of the affected limbs. The skin colour was normal and the activity was improved. Patients demonstrated sensory recovery grade of $\mathrm{S}^{3+}\left(\mathrm{S}^{0}-\mathrm{S}^{4}\right.$ grade) and two-point discrimination of $4.5 \mathrm{~mm}(3-8 \mathrm{~mm})$.

\section{Therapeutic effective evaluation}

According to the functional evaluation of replantation of severed limbs by the hand surgery branch of the Chinese Medical Association, the therapeutic scores of patients are listed in Table II. The results showed that there were 5 cases with well effect,

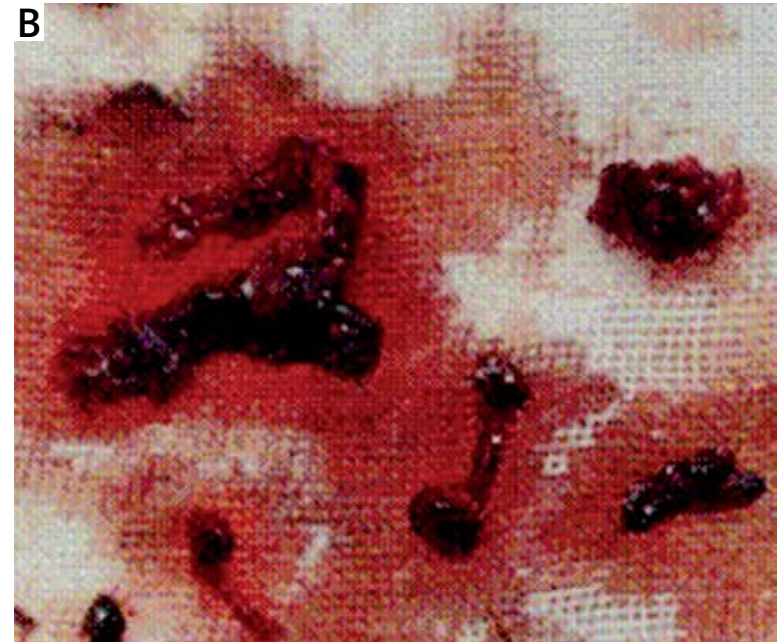

Photo 3. Popliteal vein images before and after operation. A - DSA image for the popliteal vein before treatment. B - Thrombosis removed from the popliteal vein. $\mathbf{C}$ - DSA image for the recanalisation of popliteal vein

4 cases with 4 good effect, and 2 cases with poor effect.

\section{Discussion}

In recent years, the application of DSA combined with double-chamber Fogarty balloon catheter in the field of vascular surgery is of great significance to reduce the amputation rate and mortality $[10,11]$. However, there are few reports on the application of this method in the treatment of venous crisis post replantation of severed limbs. If DSA combined with double-chamber Fogarty balloon catheter is applied in venous crisis after replantation of severed limbs, colour Doppler ultrasound should be carried out before the operation to determine the number, site, type of thrombus, and the quality of peripheral tissue of blood vessels. The treatment process 

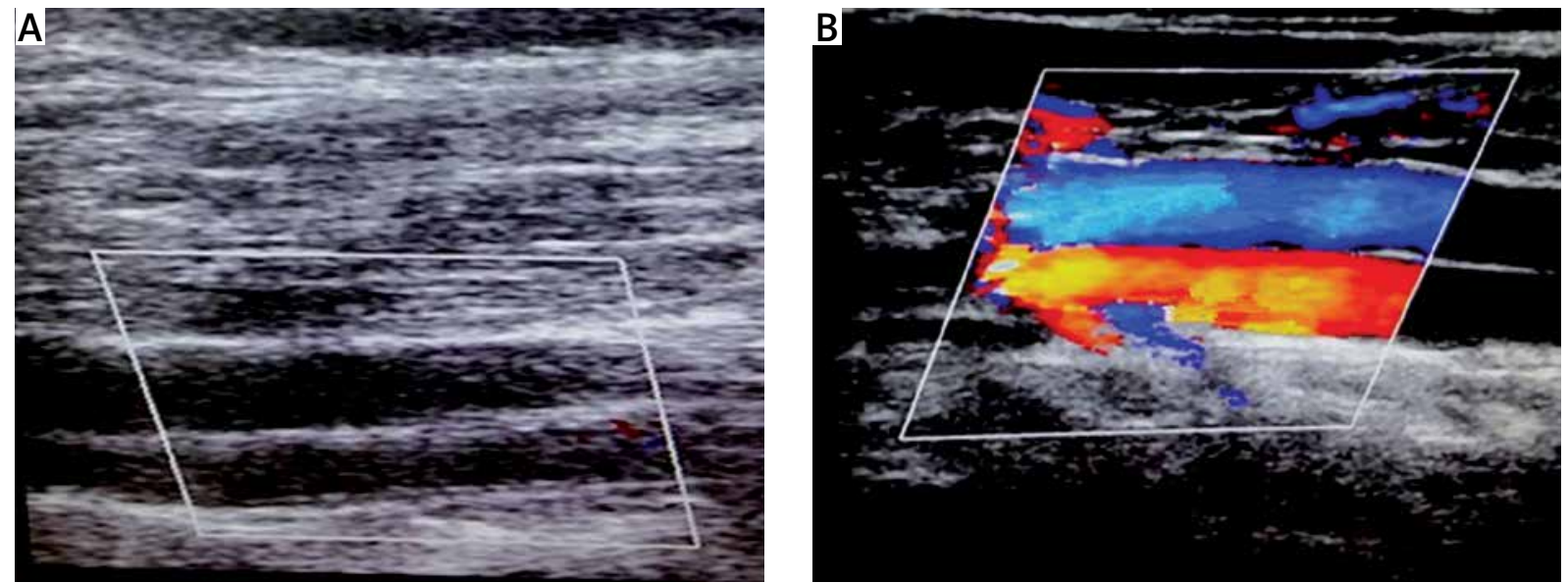

Photo 4. A typical case of a 16-year-old patient who underwent limb replantation. A - The venous crisis was formed post-operation. B - The postoperative colour Doppler ultrasound findings indicating recanalisation of the popliteal vein
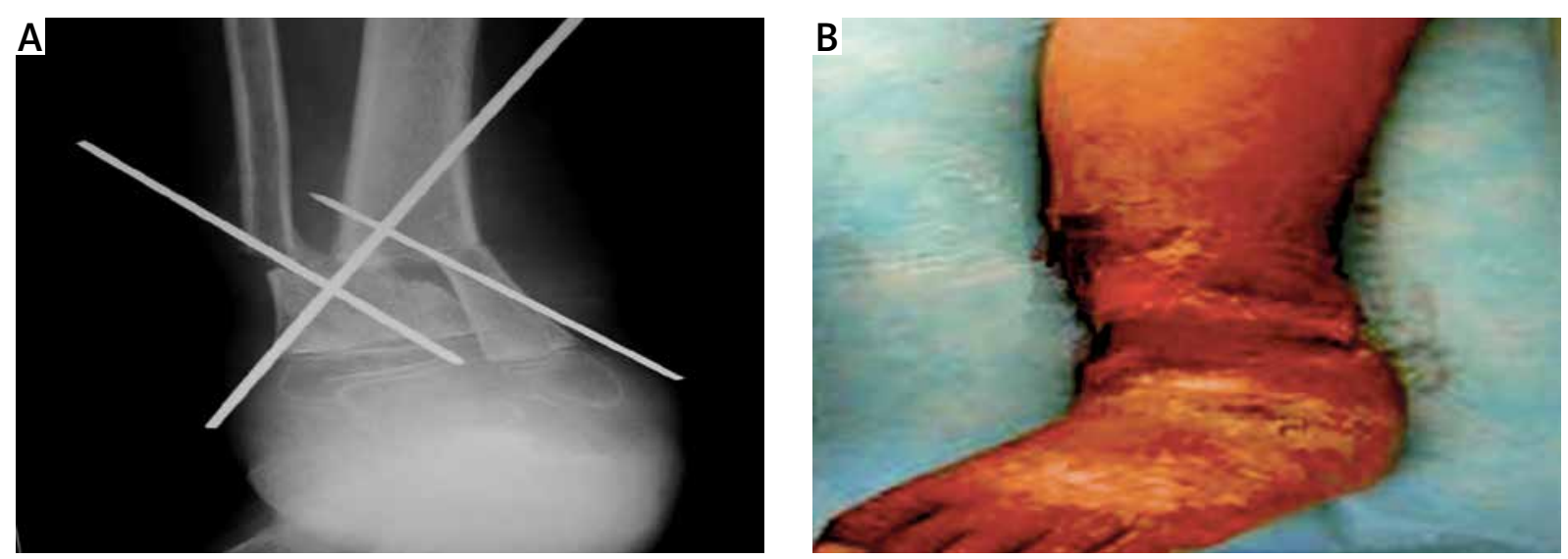

Photo 5. Postoperative condition of patients. A - Postoperative X-ray image for the patient. B - The surviving patients after the operation

requires four key steps: thrombectomy, anticoagulation, thrombolysis, and re-canalisation. Vein crisis after replantation of severed limbs is mostly caused by trauma [13].

Clinically, venous crisis is a common kind of vascular crisis after replantation of limbs. Once there is a venous crisis, it should be firstly determined whether there is compression. If there is compression, it must be removed, and the affected limb should be pad to facilitate the venous return. Meanwhile, papaverine, alprostadil injection, and other drugs are used to alleviate the symptoms. If the above conservative treatment is not relieved, we should actively explore the operation, delay the process of tissue necrosis [14], and anastomose the blood vessels again. However, the traditional open exploration operation only improves the area of the broken end of the blood vessel, which cannot resolve the situation of distal end of blood vessel embolism, and causes a second blow to the repaired area of the broken end, which is not satisfied with the effect of the combination of thrombus and non-anastomotic thrombus. Therefore, a new technique is needed to solve the above problems. In this study, DSA combined with double-chamber Fogarty balloon catheter was administrated to treat venous crisis.

The results of long-term follow-up showed that the operation was suitable for the occurrence of venous thrombosis after replantation of severed limbs. Under the guidance of DSA, Fogarty balloon catheter treatment has the following advantages: 1) It is timely, minimally invasive, effective, and has a wide range of 
Table II. Therapeutic effective evaluation scores of each item of 11 patients

\begin{tabular}{|c|c|c|c|c|c|c|c|}
\hline Cases & $\begin{array}{l}\text { Joint active } \\
\text { activity }\end{array}$ & $\begin{array}{l}\text { Muscle } \\
\text { strength }\end{array}$ & Feel & Appearance & $\begin{array}{l}\text { Residual } \\
\text { symptoms }\end{array}$ & $\begin{array}{c}\text { Work } \\
\text { situation }\end{array}$ & Total \\
\hline 1 & 26 & 17 & 16 & 8 & 7 & 7 & 81 \\
\hline 2 & 22 & 10 & 14 & 5 & 7 & 7 & 65 \\
\hline 3 & 20 & 12 & 13 & 7 & 7 & 7 & 66 \\
\hline 4 & 28 & 18 & 17 & 9 & 10 & 10 & 92 \\
\hline 5 & 10 & 5 & 5 & 5 & 5 & 5 & 35 \\
\hline 6 & 25 & 9 & 7 & 8 & 6 & 6 & 61 \\
\hline 7 & 28 & 16 & 18 & 8 & 10 & 7 & 87 \\
\hline 8 & 26 & 15 & 15 & 9 & 7 & 7 & 79 \\
\hline 9 & 28 & 19 & 17 & 10 & 10 & 10 & 94 \\
\hline 10 & 5 & 4 & 4 & 5 & 4 & 3 & 25 \\
\hline 11 & 28 & 19 & 17 & 10 & 10 & 10 & 94 \\
\hline
\end{tabular}

action areas [13], which can shorten the time of limb ischaemia to the greatest extent and reduce the rate of re-embolism. The systemic negative effect was small, the risk of bleeding was significantly reduced, and the therapeutic effect was significantly improved. There were no bleeding symptoms in intracranial, fundus, and digestive tract. 2) The double-chamber Fogarty balloon catheter improved the compliance, safety, and pertinence of the catheters, and the re-canalisation rate was significantly increased. 3) Under the guidance of DSA, the thrombus location of the double lumen Fogarty catheter is more accurate [15], which can detect thrombus in a timely manner, deal with it quickly, and reduce intra-operative haemorrhage. At the same time, the application of real-time angiography re-examination after the operation showed that the remaining thrombus was found, and the rate of re-embolism was significantly reduced after active treatment, so as to ensure the complete patency of the whole vein vascular network [16] and improve the operation effect.

In this study, patients were carefully asked about their previous medical history before operation, and colour Doppler ultrasound was performed to evaluate their condition comprehensively. At the same time, colour Doppler ultrasound was used to determine whether the patients could tolerate anticoagulation and thrombolysis. During the operation, the Fogarty catheter should be operated gently to avoid intimal injury and vascular rupture. Postoperative complications such as myopathy, nephrotic metabolic syndrome, renal failure, osteofascial compartment syndrome, circulatory failure, and death may occur.
In this study, 1 patient suffered from acute liver function damage after operation. The injured limb was completely severed due to smashing and pressing injury. The limb had a lot of trauma and tissue necrosis. Therefore, patients and their families must be informed of the risks, complications, and prognosis of the operation. The main complication is the recurrence of vascular crisis. It is suggested that the open exploration and repair should be changed.

In this study, the excellent rate was $45.5 \%$ and good rate was $36.4 \%$; therefore, the treatment effect was satisfactory. Clinically, the DSA combined with double-chamber Fogarty balloon catheter mainly applies to the patients diagnosed as " $Y$ " type vascular and venous crisis thrombus by colour Doppler ultrasound after limb replantation and the patients who can tolerate Fogarty balloon catheter thrombectomy and anticoagulation/thrombolysis by coagulation factor and colour Doppler ultrasound before operation. The above indications must be strictly controlled. Vein crisis occurs after replantation of the severed limb, but no vein thrombus is found in ultrasonic examination. It is considered as venous spasm, not the indication of the operation. If the catheter is used, it will aggravate the side injury of the diseased vessels, lead to the stubborn spasm and embolism of the vein vessels, and to limb necrosis. Before the operation, if the patients are found to have serious basic heart diseases (such as atrial fibrillation, large area myocardial infarction, etc.) by colour Doppler ultrasound, or the patients with severe coagulation disorders by coagulation factor examination, or the 
existence of active bleeding is clear, the patients who cannot tolerate anticoagulation and thrombolysis are not the indications of the operation.

In this study, the advantages of DSA combined with double-chamber Fogarty balloon catheter operation are obvious. Firstly, it has the advantages of being minimally invasive, fast, less traumatic, with fewer complications, and shorter recovery time [15]. With the deepening of the catheter in the diseased vessels, venous thrombosis can be treated just once and quickly to avoid serious tissue damage caused by long-term blockage of the thrombus [16]. Secondarily, guided by digital subtraction angiography technology, the operation of the catheter in the diseased blood vessels is safer, and the location of the thrombus is accurately located. Finally, the double-chamber Fogarty balloon catheter is used to treat the diseased blood vessels and branches, so as to avoid the blindness and experience of the operation, so that the re-embolism rate of the vein is significantly reduced, and the re-canalisation rate is obviously increased.

There are also limitations in this study: 1) Both doctors and patients are subjected to radiation. 2) The hospital must have a catheter room with operating conditions, and should not rush to perform the operation. 3) The application of catheter and balloon will inevitably increase the burden of patients. 4) The number of samples in this study is small, so we need to accumulate cases to further increase clinical experience. All of the above limitations would be improved for promoting the efficacy of double-chamber Fogarty balloon catheter operation in subsequent studies.

\section{Conclusions}

DSA combined with double-chamber Fogarty balloon catheter was used in the treatment of venous crisis, which demonstrated the accurate location of thrombus, the improved pertinence of vein branches, and satisfactory clinical effect, showing itself to be a minimally invasive and fast approach.

\section{Conflict of interest}

The authors declare no conflict of interest.

\section{References}

1. Aman M, Forssblad M, Larsen K. Incidence and body location of reported acute sport injuries in seven sports using a national insurance database. Scand J Med Sci Sports 2018; 28: 1147-58.
2. Chai Y. The development and present situation of limb replanting and reconstruction in China. Zhongguo Xiu Fu Chong Jian Wai Ke Za Zhi 2018; 32: 798-802.

3. Chen H, Zhu Z, Xu G. Experiment and clinical application of squeezing technique in venous crisis after replantation of finger. Zhongguo Xiu Fu Chong Jian Wai Ke Za Zhi 1997; 11: 347-9.

4. Xia CD, Di HP, Xue JD, et al. Effects of free anterolateral femoral or medical calf flaps in the repair of severe facial burns. Zhonghua Shao Shang Za Zhi 2019; 35: 512-6.

5. Asif A, Bakr MM, Levitt M, et al. Best vascular access in the elderly: time for innovation? Blood Purif 2019; 47: 236-9.

6. Manetta F, Newman J, Mattia A. Indicators for thoracic endovascular aortic repair (TEVAR): a brief review. Int J Angiol 2018; 27: 177-84.

7. Zhang K, Zhang SG, Jiang Y, et al. Laparoscopic hepatic left lateral lobectomy combined with fiber choledochoscopic exploration of the common the bile duct and traditional open operation. World J Gastroenterol 2018; 14: 1133-6.

8. Rhodes M, Cho JS, Gloviczki P, et al. Thrombolysis for experimental deep venous thrombosis maintains valvular competence and vasoreactivity. J Vasc Surg 2000; 31: 1193-205.

9. Brzegowy P, Kucybala I, Krupa K, et al. Angiographic and clinical results of anterior communicating artery aneurysm endovascular treatment. Videosurgery Miniinv 2019; 14: 451-60.

10. Thiex R, Moller-Hartmann W, Hans FJ, et al. Are the configuration and neck morphology of experimental aneurysms predictable? A technical approach. Neuroradiology 2004; 46: 571-6.

11. Thiex R, Hans FJ, Krings T, et al. Haemorrhagic tracheal necrosis as a lethal complication of an aneurysm model in rabbits via endoluminal incubation with elastase. Acta Neurochir 2004; 146: 285-9.

12. Pan DD, Gu YD, Si D, et al. Evaluation standard of upper limb function of Hand Surgery Society of Chinese Medical Association. Chin J Hand Surg 2000; 16: 130-5.

13. Li X, Wang P, Yu C, et al. Clinical application of ultrasound guided Fogarty balloon catheter in arterial crisis. Zhongguo Xiu Fu Chong Jian Wai Ke Za Zhi 2017; 31: 1220-4.

14. Wong AK, Joanna Nguyen T, Peric M, et al. Analysis of the risk factors associated with microvascular free flap failure using a multi-institutional database. Microsurgery 2015; 35: 6-12.

15. De DG, Setacci F, Sirignano P, et al. The combination of surgical embolectomy and endovascular techniques may improve outcomes of patients with acute lower limb ischemia. Vascular Surg 2014; 5: 729-36.

16. Greiner C, Wassmann H, Palkovic S, et al. Revascularization procedures in internal carotid artery pseudo-occlusion. Acta Neurochir (Wien) 2004; 146: 237-43.

Received: 11.09.2020, accepted: 11.10.2020. 